\title{
PROSPECTS FOR THE METHODS OF RADIONUCLIDE PRODUCTION
}

\author{
Karamian S.A. \\ Joint Institute for Nuclear Research, Dubna, Russia \\ E-mail: karamian@nrmail.jinr.ru
}

Over recent decades, the nuclear medicine is formed as a separate branch of the nuclear science. Among a variety of methods, the treatment of oncology events with proton and heavy- ion beams seems the most efficient way, but too expensive for the wide application, as well. Production and use of the radionuclide sources of radiation is definitely more economic, much lower in expenses as compared to the proton-synchrotron therapy, especially in account of the cost for the construction and infrastructure developments. The great number of radionuclides is known that allows a flexible choice of the concrete isotope and its production method individually for some definite treatment task. In literature, there are typically distinguished the therapeutic and theragnostic sources: the first ones serve for elimination of tumors due to the irradiation (external or internal), and second ones - for tomography diagnostics. The radionuclides for application in both modes are listed and characterized in the review article [1]. Various scientific and practical issues are addressed there to the production methods, to chemical processing of the active species, and even to the pharmaceutical preparations. Some cases of the "dual-purpose" isotopes are described in [1]. In the present report, only the methods of radionuclide production are stressed especially of those based on the application of low-energy electron accelerators, at $E_{e} \leq 25 \mathrm{MeV}$. After the electron-beam conversion, an intense flux of bremsstrahlung could be created, and the photon induced reactions supply a great yield for many isotopes under selective choice of the target material for concrete-species production. Another, relatively inexpensive method involves the alpha-particle beams achievable at the moderate-energy cyclotrons with $E_{\alpha} \leq 10 \mathrm{MeV} / \mathrm{amu}$. Both kind accelerators are available at FLNR, Dubna, and the discussed methods could be developed for practical uses without significant investment of funds. Possibilities are described for production of isotopes applicable for radiotherapy of patients with isotopes emitting the low-energy electromagnetic radiation and also efficient for diagnostics with the positron-emission tomography (PET) or the single photon-emission computer tomography (SPECT). Isotope production with commonly-used economic accelerators is under scope. Innovating procedures are here proposed for optimization of the production methods, as follows: a) Method of the noble-gas target providing the fast transport and separation of definite isotopes; b) New variants of the "generator method" allowing chemical separation of the product from the target material; c) Advantages of the concrete isomeric species for careful treatment of the body tissues with soft $\beta-, \gamma-$, and Auger-electron radiation; d) Possibilities for studies of the special-element metabolism "in vivo" at low-mass content of the injected element. In general, this report is mostly addressed to the nuclear science and lower to the medicine.

1. S.C.Srivastava, L.F.Mausner. Medical Radiology. Radiation Oncology chapter. Berlin, Heidelberg: Springer-Verlag, 2013, DOI: 10.1007/174_2012_782. 